Check for updates

Cite this: RSC Adv., 2018, 8, 42337

Received 30th September 2018 Accepted 7th December 2018

DOI: 10.1039/c8ra08119k

rsc.li/rsc-advances

\section{Structural design of polyurethane/poly(butylene succinate)/polycaprolactone compounds via a multilayer-assembled strategy: achieving tunable triple-shape memory performances $\dagger$}

\begin{abstract}
Yu Zheng, (D) Xiaoying Ji, Qingwen Wang, Jiabin Shen (D)* and Shaoyun Guo*
Shape memory polymeric materials composed of thermoplastic polyurethane (TPU), poly(butylene succinate) (PBS), and polycaprolactone (PCL) were prepared through layer-multiplying co-extrusion of blends. With the two well-separated melting transitions respectively from PCL and PBS, the multicomponent compounds realized triple-shape memory effect. By tailoring the structure of the individual layer, the multilayer systems with designable phase morphology were fabricated, which developed tunable shape memory performances. Particularly, the multi-continuous structure containing alternating layers of TPU and the co-continuous PBS/PCL blend presented the optimal shape fixity and recovery ratios, whose minimum value can reach $84 \%$. It was revealed that the high phase continuity along the deformation direction and the strong interfacial shearing effect promoted the ability to fix the temporary shapes and recover them to the permanent state. In addition, the multi-continuous structure maximized the synergy of the components in mechanical properties, resulting in an excellent balance of tensile strength and ductility. The present strategy for morphology control of multicomponent structures provides a promising method to fabricate outstanding triple-shape memory polymers with optimal mechanical properties.
\end{abstract}

\section{Introduction}

Shape memory polymers (SMPs), as a kind of smart materials, can provide the ability to fix a deformed shape and recover to the original shape in response to an external stimulus. ${ }^{1-6}$ In recent years, the field of SMPs has witnessed a fast growth because of the intrinsic versatility of SMPs in versatile applications ranging from sensors to aerospace structures and medical devices. ${ }^{7-11}$ For further extending the capacity of SMPs, numerous SMPs with novel properties have been developed and the triple-shape memory polymer (TSMP) is the typical one. Unlike the classic dual-SMPs that memorize only one temporary shape in a thermomechanical cycle, ${ }^{12}$ TSMPs can fix two temporary shapes and recover to the original shape by continuous heating, ${ }^{13-16}$ which meet the requirement of more complex deformation.

Generally, efficient methods to fabricate TSMPs include multicomponent polymers with well-separated thermal transitions and polymers with a broad phase transition. By selectively dividing the sufficiently broad thermal transition into sequence

Polymer Research Institute of Sichuan University, State Key Laboratory of Polymer Materials Engineering, Chengdu, Sichuan 610065, P. R. China. E-mail: shenjb@scu. edu.cn; nic7702@scu.edu.cn

$\dagger$ Electronic supplementary information (ESI) available: SEM pictures, DSC heating curves, calculated crystallization degrees, stress-strain curves. See DOI: 10.1039/c8ra08119k domains, some polymers can exhibit triple-shape memory effect (TSME). For instance, Xie found that the pure perfluorosulphonic acid (PSFA) ionomer possessing a broad glass transition can present TSME and even multiple-shape memory effect. ${ }^{17}$ In another work, Samuel et al. prepared a TSMP by simply blending the miscible poly(methyl methacrylate) (PMMA) and poly(L-lactide acid) (PLLA) to broaden the glass transition temperature range, and tunable TSME was achieved for the blend with $50 \mathrm{wt} \%$ PLLA via tuning the intermediate shapes. ${ }^{18}$ However, the chain relaxation of this type of TSMP occurs at any temperature within the broad thermal transition, which usually leads to unstable temporary shapes. Besides, the choice of the thermomechanical conditions would dramatically influence the final TSME, ${ }^{19}$ so that more care should be taken to optimize the performance.

In contrast, the multicomponent polymers realize TSME based on the distinct elastic modulus status resulted from the well-separated thermal transitions, which can make it more efficient to set the thermomechanical conditions and fix the temporary shapes. ${ }^{20}$ As an example, Behl et al. synthesised a TSMP network using PCL and poly(cyclohexyl methacrylate) (PCHMA). ${ }^{21}$ The switching temperature $\left(T_{\mathrm{s}}\right)$ at different shape memory stages can be easily selected according to the melting transition of PCL and the glass transition of PCHMA. Moreover, both of the shape fixity ratio $\left(R_{\mathrm{f}}\right)$ and shape recovery ratio $\left(R_{\mathrm{r}}\right)$ 
approximated $100 \%$ when the PCL content was between 35 and $60 \mathrm{wt} \%$. After that, the same group developed TSME by fabricating another copolymer network composed of poly( $\omega$-pentadecalactone) (PPD) and PCL with separated $T_{\mathrm{m}}{ }^{\prime} \mathrm{s} .{ }^{22}$ Although many works have been accomplished, ${ }^{23-26}$ the fabrication methods still need improvement and development.

Polymer blending, which can integrate the advantages of two or more materials, has been the rising star in fabrication of SMPs because of its easy processability. ${ }^{27,28}$ Some reports have stated that the dual-shape memory effect can be easily achieved by blending one permanent component (PC) which is in charge of memorizing the permanent shape and another switching component (SC) with reversible phase transition. ${ }^{29-32}$ Inspired by that, incorporating additional SC's through melt processing seems like an effective route to develop TSMPs. By so far, some multi-phase blends possessing TSME have been reported, such as polyethylene (PE)/polycyclooctene blends and thermoplastic polyurethane (TPU)/olefin block copolymer/PCL blends. ${ }^{33,34}$ For the shape memory compounds, the co-continuous morphology was known to show better shape memory performance. ${ }^{\mathbf{2 0}, 35}$ However, it is hard to tailor a multicomponent structure to be co-continuous via conventional melt processing because of the numerous influencing factors (e.g. viscosity ratios, interfacial tensions, processing parameters, and so on). ${ }^{36-38}$
The layer-multiplying co-extrusion, as an advanced processing technology, has attracted tremendous attention due to its application in developing functional materials with versatile unique properties..$^{39-45}$ As schematically illustrated in Fig. 1, the multilayer structure possessing a special co-continuous morphology can be prepared through combining an assembly of layer-multiplying elements (LMEs) with two extruders, regardless of the influencing factors proposed in the blending system. Moreover, the structure of the individual layer which may be a blend can be tailored before the layer-assembly process, thus being beneficial for incorporating more SC's, meanwhile, achieving morphology control. Besides, the Baer's group (Case Western Reserve University) has reported that the high phase continuity and numerous interfaces of multilayer SMPs would greatly facilitate stress transferring and load distribution, which maximizes synergies among the shape memory domains. ${ }^{\mathbf{4}, 47}$ Accordingly, the layer-multiplying coextrusion that enables the structural design of the multicomponent system to become controllable and efficient is believed to be a promising strategy for preparing excellent TSMPs.

In this work, multicomponent structures with tunable phase continuity were fabricated through layer-multiplying coextrusion. The poly(butylene succinate) (PBS)/PCL blend with co-continuous structure was coextruded with neat TPU, for

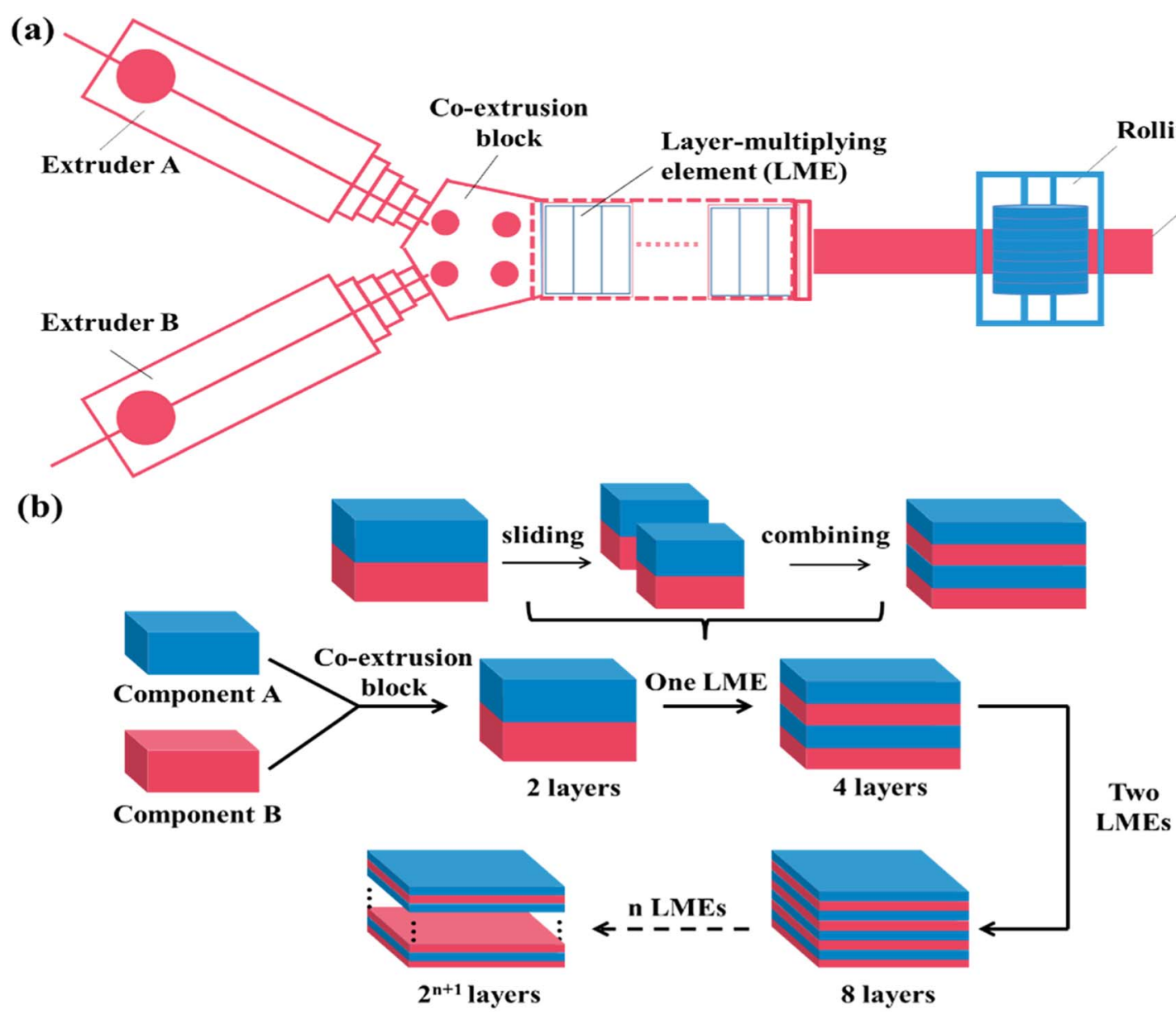

Fig. 1 Schematic of the (a) layer-multiplying co-extrusion system and (b) production progress of multilayer structure. 
formation of a special multi-continuous morphology. Additionally, another multilayer structure with continuous PCL and dispersed PBS was prepared by coextruding the co-continuous TPU/PCL blend and the sea-island TPU/PBS blend. TSME of the multilayer systems was investigated and the operative shape memory mechanism related to the phase continuity was discussed. The influence of the morphological distinctions on mechanical properties was also explored. Furthermore, the materials used in this work have excellent biocompatibility, ${ }^{32,48,49}$ so that the multilayer TSMP is of great potential in biomedical applications.

\section{Experimental}

\subsection{Materials}

Polyester-based TPU (Elastogran ${ }^{\circledR}$ S85A11), with a Shore A hardness of about 85 , a density of $1.23 \mathrm{~g} \mathrm{~cm}^{-3}$, and $4,4^{\prime}$ methane diisocyanate (MDI) acting as the hard segments, was purchased from BASF group (Germany). Commercial PCL (CAPA6800) with a density of $1.11 \mathrm{~g} \mathrm{~cm}^{-3}$ was purchased from Perstorp Corp. (UK). PBS (1001MD) with a density of $1.26 \mathrm{~g} \mathrm{~cm}^{-3}$ was purchased from Showa Denko Corp. (Japan). The molecular structures of the materials are illustrated in Fig. 2.

\subsection{Specimen preparation}

TPU and PBS were dried at $80{ }^{\circ} \mathrm{C}$ for $40 \mathrm{~h}$ and PCL was dried at $40{ }^{\circ} \mathrm{C}$ for $24 \mathrm{~h}$ in a vacuum oven before melt processing. After that, the PBS/PCL, TPU/PBS, and TPU/PCL blend pellets (denoted as SLB, TSB, and TLB) respectively contained $50 \mathrm{vol} \%$ PCL, 50 vol\% PBS and 50 vol\% PCL were prepared using a SHJ20 twin-screw extruder (screw diameter is $21.7 \mathrm{~mm}, L / D=40: 1$, Nanjing Giant Co. Ltd., China) with an extrusion temperature of $185^{\circ} \mathrm{C}$ and extrusion rate of $150 \mathrm{rpm}$. Then, the multilayer SMPs consisting of alternating TPU and SLB layers were prepared through the layer-multiplying co-extrusion technology. As

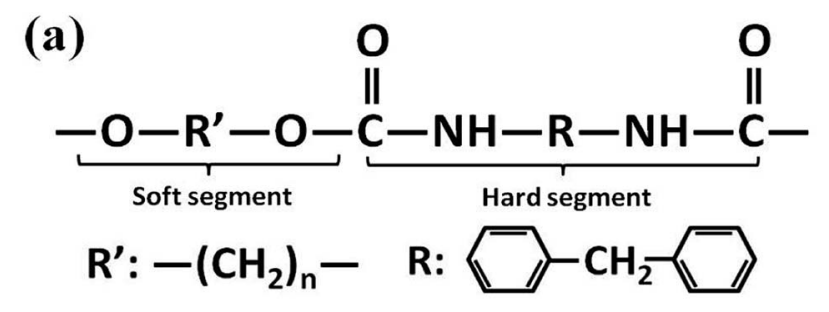

(b)

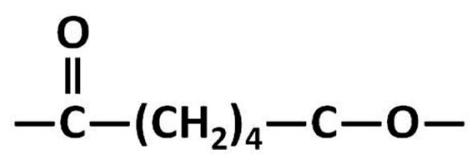

(c)

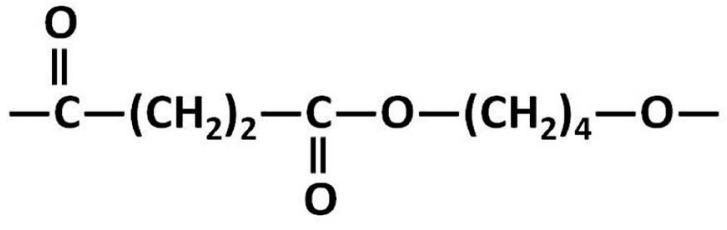

Fig. 2 Molecular structures of (a) TPU, (b) PCL and (c) PBS. shown in Fig. 1a, TPU and SLB were simultaneously extruded from different extruders, combined as a two-layer melt in the coextrusion block, and then flowed through an assembly of LMEs. In a LME, the melt was sliced into two left and right sections by a divider and then recombined vertically, leading to double the number of layers, Fig. 1b. When $n$ LMEs were applied, the materials with $2^{(n+1)}$ layers can be produced eventually, while the overall thickness of the multilayers remain unchanged. In this work, 64-layer material was fabricated by applying 5 LMEs. By controlling the co-extrusion speed, the total thickness of the extrudate was maintained at about $1.0 \mathrm{~mm}$, and the thickness ratio of the TPU and SLB layers was kept at $1: 1$. The processing conditions were the same as those for the preparation of SLB pellets. Similarly, the 64-layer TSB/TLB specimen with the whole thickness of $1.0 \mathrm{~mm}$ was produced under the same conditions. What should be noted is that the thickness ratio of TSB and TLB is also $1: 1$, in order to maintain the composition unchanged. For comparison, TPU/PBS/PCL blend (donated as TSLB) with the same composition as that in the multilayer systems was produced by extruding from one of extruders of above coextrusion system, for keeping a similar processing history.

\subsection{Differential scanning calorimetry (DSC)}

For determining the $T_{\mathrm{s}}$ at the different shape memory stages, the heating and cooling curves of the samples were recorded on a DSC (Q20, TA Instrument, USA) under nitrogen. Each 5-7 mg sample was heated from 20 to $160{ }^{\circ} \mathrm{C}$ at a heating rate of $10{ }^{\circ} \mathrm{C} \min ^{-1}$ and maintained at $160{ }^{\circ} \mathrm{C}$ for $5 \mathrm{~min}$, then the sample was cooled down to $0{ }^{\circ} \mathrm{C}$ at a cooling rate of $10^{\circ} \mathrm{C} \mathrm{min}{ }^{-1}$.

The degree of crystallizations $\left(X_{\mathrm{c}}\right)$ of PCL and PBS in each sample was calculated as follows:

$$
X_{\mathrm{c}}=\frac{\Delta H_{\mathrm{m}}}{\Delta H_{\mathrm{m}}^{0} \times W_{\mathrm{x}}} \times 100 \%
$$

where $\Delta H_{\mathrm{m}}$ is the enthalpy of PCL or PBS, which was obtained from the DSC heating curve by integral computation of the melting peak using TA Universal Analysis software; $\Delta H_{\mathrm{m}}^{0}$ is the enthalpy of $100 \%$ crystallinity of PCL and PBS, which are 135.5 and $110.3 \mathrm{~J}$, respectively; ${ }^{50,51} W_{\mathrm{x}}$ is the weight fraction of PCL or PBS in each sample, which can be obtained based on the densities and compositions of the components.

\subsection{Morphological observation}

The microstructure of each specimen was observed using an Olympus BX51 polarizing light microscope (PLM) equipped with a camera. A thin slice about $10 \mu \mathrm{m}$ in thickness was obtained by a microtome from each sample perpendicular to the extruding direction.

The morphological structure of the individual layer was further observed by using a scanning electron microscope (SEM, JEOL JSM-5900LV) under a proper accelerating voltage. The specimens were first cryofractured in liquid nitrogen, then SLB and TLB were immersed in acetone to selectively etch the dispersed PCL, TSB and TSLB were immersed in dichloromethane to selectively etch the dispersed PBS or/and PCL. Prior 
to visualization, the surface of each specimen was coated with a layer of gold in a vacuum chamber.

\subsection{Shape memory test}

The samples for shape memory testing were cut from the extruded films into rectangular specimens with dimensions of $10 \mathrm{~mm}$ (length) $\times 2 \mathrm{~mm}$ (width) $\times 1.0 \mathrm{~mm}$ (thickness). TSME experiments were carried out on the dynamic mechanical analyzer (Q800, TA Instrument) in controlled force mode. A typical thermomechanical cycle involves the following steps: (1) stretching the sample at $T_{\mathrm{s} 1}$ to a certain strain $\left(\varepsilon_{\mathrm{m} 1}\right)$ with a constant stress, (2) cooling to a lower $T_{\mathrm{s} 2}$ and then removing the load for 5 min to obtain the first temporary strain $\left(\varepsilon_{\mathbf{f} 1}\right)$, (3) a second force-controlled stretching was performed at $T_{\mathrm{s} 2}$ to reach the maximum strain $\left(\varepsilon_{\mathrm{m} 2}\right)$, (4) cooling to $25{ }^{\circ} \mathrm{C}$ and again removing the stress to gain the second temporary strain $\left(\varepsilon_{\mathrm{f} 2}\right)$, and (5) a free-strain recovery was first performed at $T_{\mathrm{s} 2}$ for $20 \mathrm{~min}$ to the recovered strain $\left(\varepsilon_{\mathrm{r} 2}\right)$, subsequently performed at $T_{\mathrm{s} 1}$ for $20 \mathrm{~min}$ to the final strain $\left(\varepsilon_{\mathrm{r} 1}\right) . R_{\mathrm{r}}$ and $R_{\mathrm{f}}$ were respectively calculated by using following eqn (1) and (2): ${ }^{17,18}$

$$
\begin{gathered}
R_{\mathrm{f} x}=\frac{\varepsilon_{\mathrm{f}(x)}}{\varepsilon_{\mathrm{m}(x)}} \times 100 \% \\
R_{\mathrm{r} x}=\frac{\varepsilon_{\mathrm{f}(x)}-\varepsilon_{\mathrm{r}(x)}}{\varepsilon_{\mathrm{f}(x)}-\varepsilon_{\mathrm{f}(x-1)}} \times 100 \%
\end{gathered}
$$

\subsection{Dynamic mechanical analysis}

The storage moduli at different temperatures were measured through the DMA Q800 from TA Instruments. Each sample with dimensions of $10 \mathrm{~mm}$ (length) $\times 4 \mathrm{~mm}$ (width) $\times 1.0 \mathrm{~mm}$ (thickness) was heated at heating rate of $3{ }^{\circ} \mathrm{C} \mathrm{min}^{-1}$ from 0 to $160{ }^{\circ} \mathrm{C}$ using a stretching mode. The testing frequency was $10 \mathrm{~Hz}$.

\subsection{Creep-recovery test}

The creep-recovery curves were measured using the DMA Q800 from TA Instruments at the $T_{\mathrm{s}}$. Each sample was first stretched to a certain strain under the constant stress and then the load was released instantaneously. The creeping process lasted for $20 \mathrm{~min}$ and the recovered strain as a function of the creeping time was recorded.

\subsection{Tensile tests}

Tensile tests were performed using an Instron 4302 tension machine (Canton, MA, USA) at a crosshead speed of 100 $\mathrm{mm} \mathrm{min}^{-1}$ in accordance with ASTM D638. At least five specimens for each sample were tested, and the average value was calculated.

\section{Results and discussion}

\subsection{Macrostructure}

Fig. 3 exhibits the PLM pictures of the blending and multilayer specimens. The parallel-assembled bright and dark layers of TPU/SLB are respectively corresponding to the semicrystalline SLB and amorphous TPU. Besides, the individual SLB demonstrates the typical co-continuous morphology, which can be proved by the SEM picture showed in Fig. S1. $\dagger$ Thus, a special multi-continuous structure with numerous interfaces can be fabricated by coextruding SLB and TPU. TSB/TLB also shows a multilayer structure composed of alternating semicrystalline layers. However, the interfaces in TSB/TLB are more ambiguous than those in TPU/SLB because of the molecular diffusion of the TPU matrix during the layer-multiplying process. Additionally, as seen in Fig. S2, $\uparrow$ TLB appears to be co-continuous, while TSB exhibits the sea-island morphology. Accordingly, TSB/TLB can be regarded as a TPU-based blend in which the continuous PCL and island-like PBS are alternately dispersed. For the conventional blend, it can be seen that the bright crystals of PBS and PCL are dispersed in the amorphous TPU matrix. By etching both of PBS and PCL with dichloromethane, ${ }^{52}$ the typical seaisland structure can be observed through the SEM test, Fig. S3.† Consequently, the multilayer assembly of different structures can produce multicomponent systems with tunable phase continuity, which is believed to result in distinct shape memory performance.

\subsection{Thermal behaviors}

As mentioned earlier, the TSME can be achieved by incorporating two reversible domains with well-separated thermal transitions. Fig. 4 displays the DSC heating and cooling curves of the blending and multilayer specimens. From Fig. 4a, it can be seen that all the curves show a similar melting transition process of PCL and have the same $T_{\mathrm{m}}$ at around $56^{\circ} \mathrm{C}$ as that of PCL, irrespective of the structural distinctions. On the other
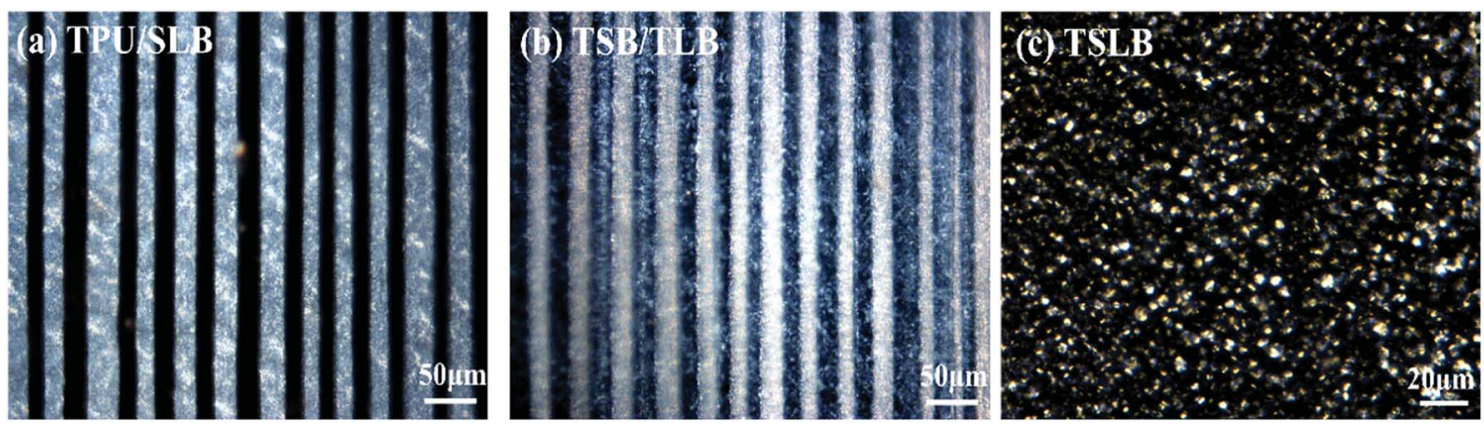

Fig. 3 PLM pictures of the (a) TPU/SLB, (b) TSB/TLB and (c) TSLB specimens. 

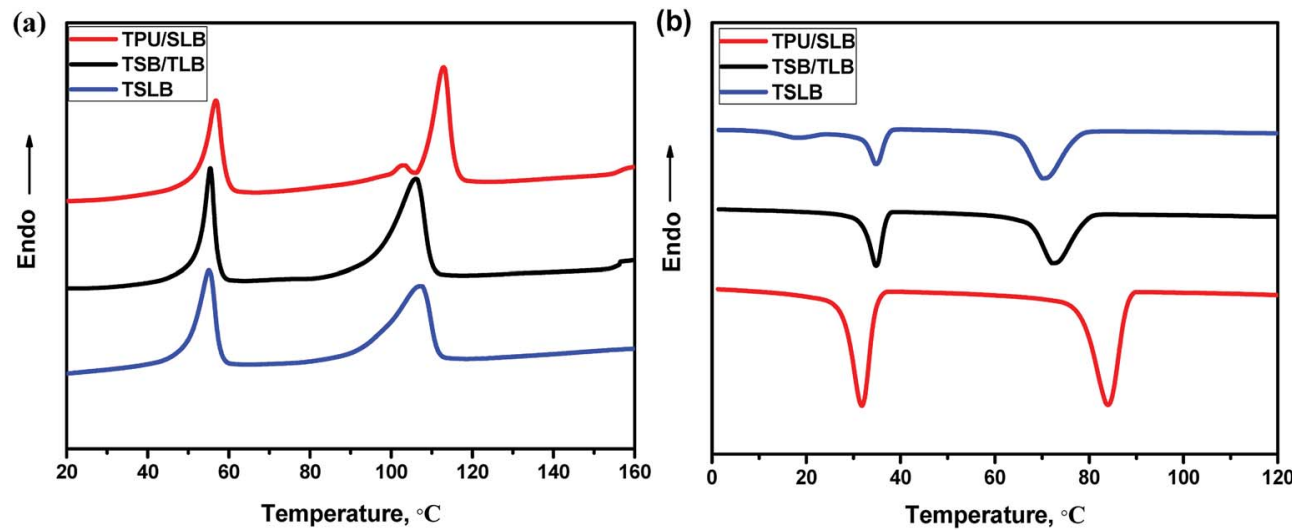

Fig. 4 DSC (a) heating and (b) cooling curves of the specimens.

hand, PBS in TPU/SLB exhibits a dual melting behavior with two $T_{\mathrm{m}}$ 's at approximately 103 and $113{ }^{\circ} \mathrm{C}$ ascribed to its meltrecrystallization effect..$^{53}$ However, both of TSB/TLB and TSLB have only a single melting peak of PBS at about $107^{\circ} \mathrm{C}$, which is basically consistent with the DSC curve of TSB exhibited in Fig. S4. $\dagger$ Unlike the TPU/SLB multilayer structure with numerous interfaces, TPU is directly blended with PBS in TSB/ TLB and TSLB. Thus, the melt-recrystallization effect of PBS may be suppressed by the hydrogen-bonding interaction between TPU and PBS..$^{54}$ Although PBS demonstrates different melting behavior, all the specimens can provide two wellseparated $T_{\mathrm{m}}$ 's to meet the prerequisite for realizing TSME.

As for a triple-shape memory cycle, the fixation of the first temporary shape $\left(\mathrm{S}_{1}\right)$ is crucial but always more difficult due to the chain mobility of the SC owning the lower phase transition temperature. In this study, when $\mathrm{S}_{1}$ was fixed at $T_{\mathrm{s} 2}$ which should be higher than the $T_{\mathrm{m}}$ of PCL to allow a second deformation, the molten PCL would cause partial recovery. Fortunately, as seen in Fig. 4b, although the crystallization temperature $\left(T_{\mathrm{c}}\right)$ of PBS would be decreased after blending with TPU, which is still appropriately higher than the $T_{\mathrm{m}}$ of PCL, thus can provide a temperature interval to choose a proper $T_{\mathrm{s} 2}$ where PBS can crystallize to maintain $S_{1}$. Similarly, after cooling the second temporary shape $\left(S_{2}\right)$ to the room temperature, the crystalline PCL would make contribution to the shape fixation. Hence, the well-separated $T_{\mathrm{c}}{ }^{\prime} \mathrm{s} / T_{\mathrm{m}}$ 's of PCL and PBS endow the specimens with great potential to demonstrate efficient TSME.

\subsection{Shape memory performance}

Based on above results of thermal behaviors, TSME of each specimen was investigated by designing a triple-shape memory cycle, as shown in Fig. 5 . The $T_{\mathrm{s} 1}$ was chosen at $125{ }^{\circ} \mathrm{C}$ where both of PBS and PCL are molten to obtain $\mathrm{S}_{1}$, and then the deformation was fixed by cooling to the $T_{\mathrm{s} 2}$ of $70{ }^{\circ} \mathrm{C}$ where PBS can crystallize to provide mechanical support and PCL is still molten to allow the second shaping. After fixing the $S_{2}$ by cooling to $25{ }^{\circ} \mathrm{C}$, a subsequent recovery under continuous heating was conducted to characterize the recoverability. The corresponding $R_{\mathrm{f}}$ and $R_{\mathrm{r}}$ at each stage are calculated and compared in Table 1 . The $R_{\mathrm{f} 2}$ and $R_{\mathrm{r} 2}$ of TSLB are only 78.2 and $66.0 \%$, respectively, suggesting that the fixation of $\mathrm{S}_{2}$ and the recovery from $S_{2}$ to $S_{1}$ are not effective. After tailoring PCL or/ and PBS to be continuous through layer-multiplying coextrusion, all the values are greatly improved. For the TSB/TLB specimen, only $R_{2}$ is relatively low, but is still around $75 \%$, and both of $R_{\mathrm{f} 1}$ and $R_{\mathrm{r} 1}$ are beyond $88 \%$. Besides, the minimum $R_{\mathrm{f}}$ or $R_{\mathrm{r}}$ value of TPU/SLB can even reach $84 \%$, although the strains in Fig. 5 show higher values for TPU/SLB specimens, which further indicates that the multi-continuous structure can indeed better promote the shape memory performances.

Fig. 6 exhibits the macroscopic triple-shape memory progress of TPU/SLB recorded by a digital camera. The stripe was first deformed to a "L" shape at $125{ }^{\circ} \mathrm{C}$ and fixed at $70{ }^{\circ} \mathrm{C}$ for 5 min to obtain $\mathrm{S}_{1}$; then it was further deformed to a square shape and cooled to $25{ }^{\circ} \mathrm{C}$ to fix $\mathrm{S}_{2}$. After that, the $\mathrm{S}_{2}$ was recovered to $\mathrm{S}_{1}$ by heating the sample to $70{ }^{\circ} \mathrm{C}$ and isothermal treatment for $10 \mathrm{~min}$. Subsequently, the sample was further heated to $125^{\circ} \mathrm{C}$ and isothermally treated for another $10 \mathrm{~min}$. It can be found that the two temporary shapes are well fixed and the original shape is almost entirely recovered. Accordingly, the TPU/SLB can indeed present a valuable TSME with excellent and balanced shape memory performances over all stages.

\subsection{Shape memory mechanism}

With regard to a multicomponent SMP, the shape memory property is always governed by the phase structure..$^{29-32}$ For this study, above results of morphological observation have revealed that different geometric organization of the components would produce diverse macrostructures as schematically illustrated in Fig. 7, resulting in distinct TSME. It can be seen that the phase continuity of PBS and PCL are gradually enhanced ranging from TSLB to TPU/SLB. Hence, the shape memory mechanism of TSME, which seems to be related to the phase continuity, is worth discussing.

When $\mathrm{S}_{1}$ was fixed at $70{ }^{\circ} \mathrm{C}$, the specimen tended to recover because TPU preserved the stain energy and PCL is molten, but most of the deformation would be maintained with the mechanical support provided by the crystallized PBS. For the TPU/SLB specimen, the continuous PBS can serve as the framework of the SLB layers, so that the recovery tendency of PCL and TPU can be well restrained. In contrast, the dispersed PBS in the TPU matrix would obviously allow more shape 

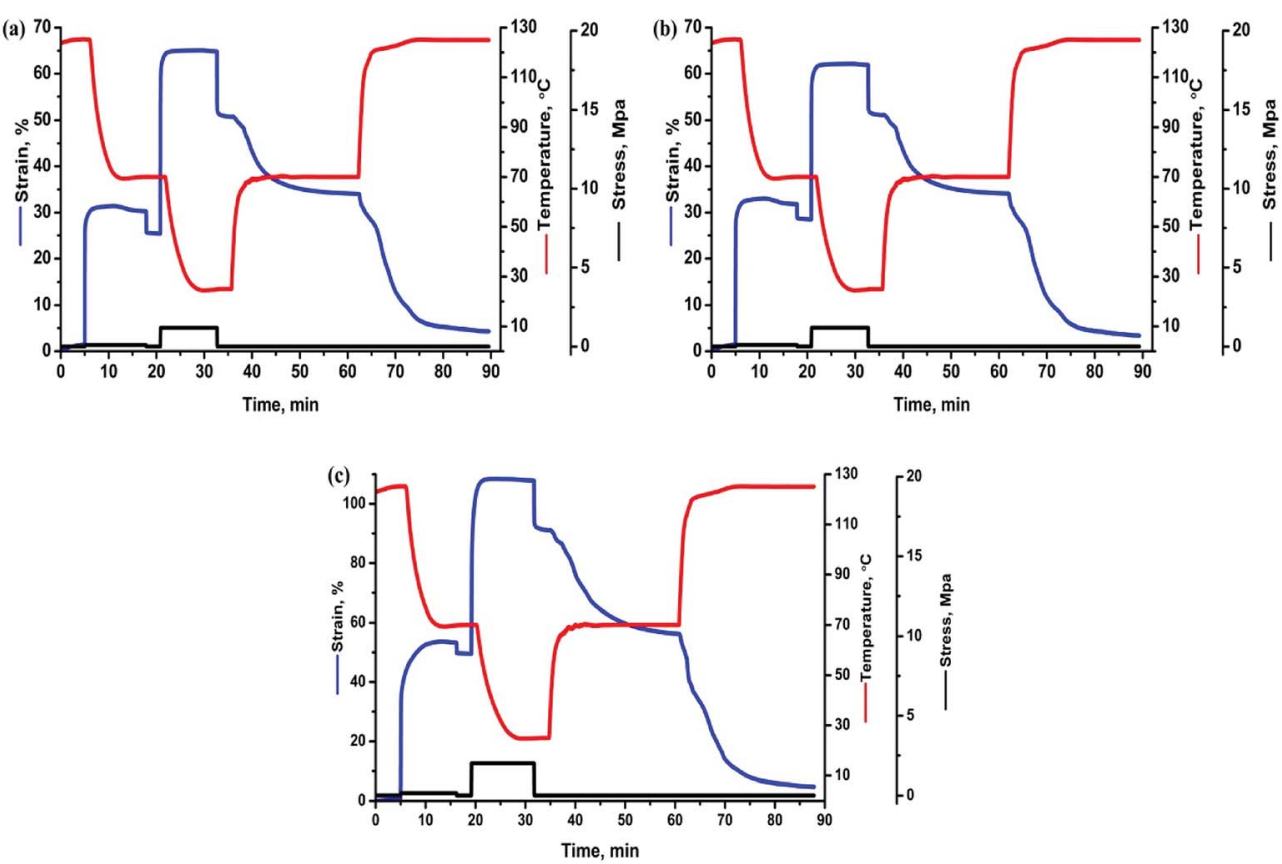

Fig. 5 The triple-shape memory cycles with $T_{s}$ 's of 70 and $125^{\circ} \mathrm{C}$ of the (a) TSLB, (b) TSB/TLB, and (c) TPU/SLB specimens.

Table $1 \quad R_{\mathrm{f}}$ and $R_{\mathrm{r}}$ values of the specimens at each stage in the tripleshape memory progress

\begin{tabular}{lllll}
\hline Sample & $R_{\mathrm{f} 1}$ & $R_{\mathrm{f} 2}$ & $R_{\mathrm{r} 2}$ & $R_{\mathrm{r} 1}$ \\
\hline TSLB & $83.8 \%$ & $78.2 \%$ & $66.0 \%$ & $83.1 \%$ \\
TSB/TLB & $89.6 \%$ & $82.6 \%$ & $75.2 \%$ & $88.1 \%$ \\
TPU/SLB & $92.9 \%$ & $84.5 \%$ & $83.9 \%$ & $90.3 \%$
\end{tabular}

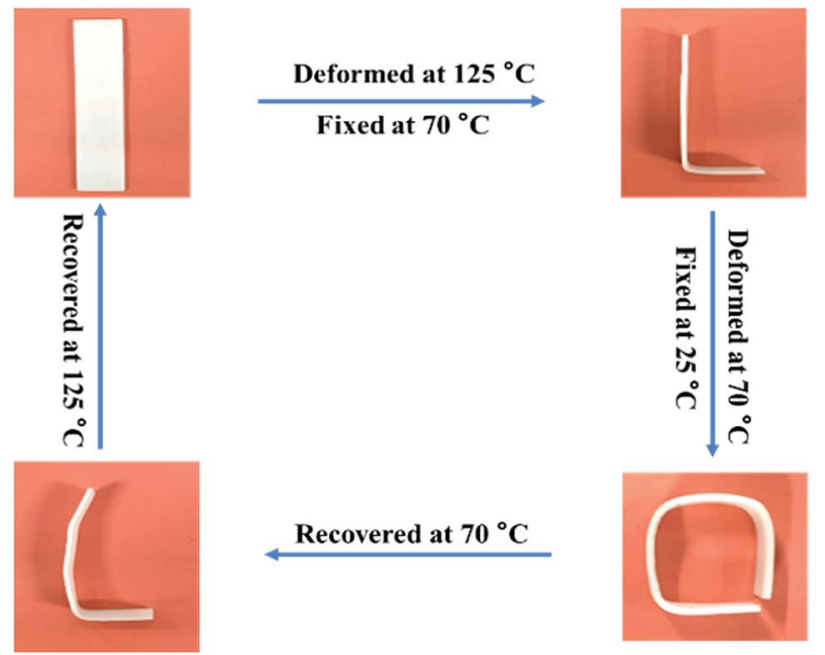

Fig. 6 The macroscopic triple-shape memory progress of the TPU/ SLB specimen.

recovery, leading to the reduction of $R_{\mathrm{f} 1}$. Moreover, the storage moduli of the specimens at $70{ }^{\circ} \mathrm{C}$ are gradually enhanced with the increase of the phase continuity of PBS, Fig. 8, further indicating that the continuous PBS can provide a stronger mechanical support to fix the temporary deformation. Similarly, as $\mathrm{S}_{2}$ was fixed at $25{ }^{\circ} \mathrm{C}$, the multilayer systems with continuous PCL demonstrate the better shape fixity. Besides, it should be noticed that the $R_{\mathrm{f} 1}$ of each specimen is larger than $R_{\mathrm{f} 2}$. Actually, $R_{\mathrm{f}}$ is significantly influenced by the amorphous domains of PBS and PCL which can induce recovery under the driving force provided by TPU due to their extremely low $T_{\mathrm{g}}$ 's of about -30 and $-60{ }^{\circ} \mathrm{C}$, respectively. However, PBS has a much higher $X_{\mathrm{c}}$ than that of PCL, Table S1, $\uparrow$ meaning that there may be more less active domains when $\mathrm{S}_{1}$ was fixed by PBS. Additionally, the specimens have a quite greater storage modulus at $70{ }^{\circ} \mathrm{C}$, which would produce larger strain energy to be stored in the second shaping progress, so that the recovering tendency would become stronger when $\mathrm{S}_{2}$ was fixed. Accordingly, the specimen exhibits a lower $R_{\mathrm{f}}$ at the second shape fixing stage.

In the recovery progress, the performance of each specimen turns to be governed by TPU because the molten PBS and PCL with an extremely low modulus would fail to store the strain energy. For the TPU/SLB specimen, the parallel-assembled SLB and TPU layers should always obtain the same strain during deformation. Thus, the TPU layers can efficiently promote the adjacent SLB layers to recover through the interfacial shearing effect. However, the TSB/TLB multilayer system has a slightly worse $R_{\mathrm{r}}$, which may be resulted from the lower phase continuity along the stretching direction and the weaker interfacial shearing effect induced by the interfacial diffusion of TPU. With regard to TSLB with a sea-island structure, it can also be divided into many parallel layers in which the components are assembled in series. On the basis of the classic viscoelastic theory, ${ }^{55}$ the strain of the series-connected components should be dominated by their own viscoelasticity, so that the recovery of TPU has less effect on that of PBS and PCL. Consequently, TSLB would produce more irreversible deformation, which can be 


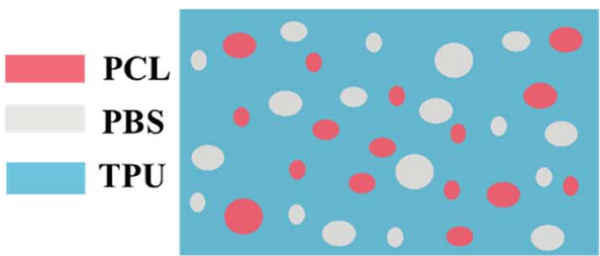

TSLB

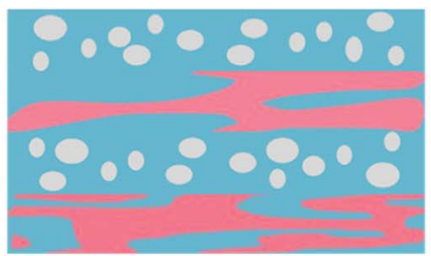

TSB/TLB

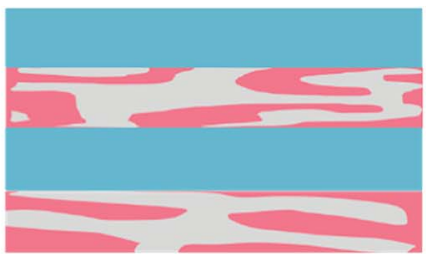

TPU/SLB

Fig. 7 Schematic of the morphological structures of the specimens.

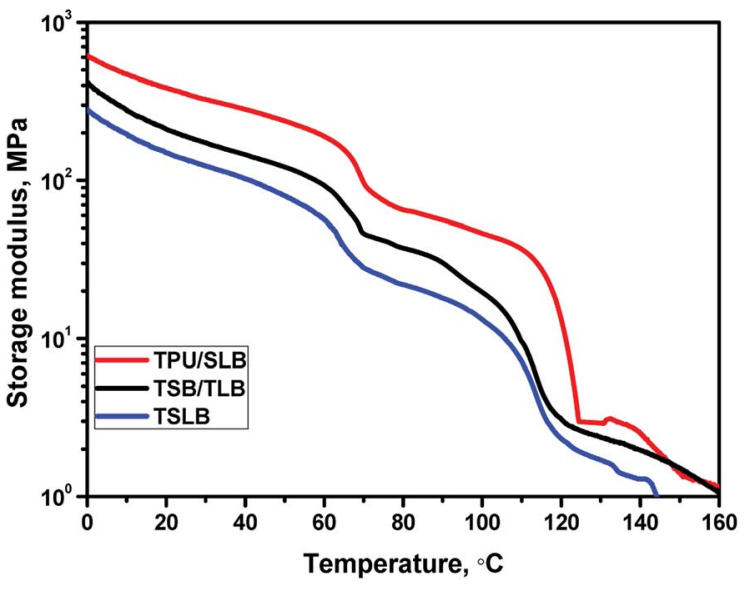

Fig. 8 The storage modulus curves of the specimens.

further evidenced through the creep-recovery experiment. Fig. 9 shows the evolution of recovered strain during the creeping progress of each specimen at the two $T_{\mathrm{s}}$ 's of 70 and $125^{\circ} \mathrm{C}$. After creeping for $20 \mathrm{~min}$ at $125{ }^{\circ} \mathrm{C}$, the TPU/SLB and TSB/TLB specimens have the recovered strains of 85.9 and $79.6 \%$, respectively, while the final strain of the blend is $76.1 \%$. When the creep temperature is decreased to $70{ }^{\circ} \mathrm{C}$, the multilayer structures also exhibit the larger recoverability than that of the blend. These results reveal that the multilayer systems with higher phase continuity and a plenty of interfaces can indeed hold a greater promise in optimizing $R_{\mathrm{r}}$. However, for the individual specimen, a lower $R_{\mathrm{r}}$ is obtained at $70^{\circ} \mathrm{C}$. Taking the TSLB specimen for instance, the $R_{\mathrm{r}}$ is dramatically decreased from 78.2 to $66.0 \%$, when the $T_{\mathrm{S}}$ is reduced from 125 to $70{ }^{\circ} \mathrm{C}$. Unlike that both of PBS and PCL would melt at the $T_{\mathrm{s} 1}$ of $125^{\circ} \mathrm{C}$,
PBS is semi-crystalline at the $T_{\mathrm{s} 2}$ of $70^{\circ} \mathrm{C}$. As a result, the second shaping progress would create some destruction and orientation of the PBS crystals which would fail to be recovered until melting again, thus deteriorating $R_{\mathrm{r}}$ after reheating to $70{ }^{\circ} \mathrm{C}$ at the first recovering stage. By further heating to $T_{\mathrm{s} 1}$, PBS would be molten to allow a full recovery, and a larger $R_{\mathrm{r}}$ is reasonably obtained.

\subsection{Mechanical properties}

Apart from promising competitive shape memory performance, favorable mechanical properties are also important for TSMPs to realize practical application. Thus, tensile tests were conducted to explore the influence of morphological distinctions among the specimens on the tensile strength $\left(\sigma_{\mathrm{s}}\right)$ and elongation at break $\left(\varepsilon_{\mathrm{b}}\right)$. The stress-strain curves and resulting data are showed in Fig. 10. With the dispersed PBS and PCL, TSLB shows the typical elastomeric tensile property with $\sigma_{\mathrm{s}}$ of $31.9 \mathrm{MPa}$ and excellent $\varepsilon_{\mathrm{b}}$ of $1274.7 \%$. By transforming PCL into the continuous phase via layer-multiplying co-extrusion, the $\sigma_{\mathrm{s}}$ and $\varepsilon_{\mathrm{b}}$ of TSB/TLB are increased to 47.2 MPa and $1496.0 \%$, respectively. However, because of the dispersed PBS, the mechanical performance of TSB/TLB is still more governed by the TPU matrix. For the TPU/SLB multilayer structure with continuous PBS and PCL, an obvious yielding behavior can be observed. Moreover, the $\sigma_{\mathrm{s}}$ is further enhanced to $53.0 \mathrm{MPa}$, but the $\varepsilon_{\mathrm{b}}$ slides to $1324.3 \%$, which is still larger than that of TSLB. This indicates that construction of a multi-continuous structure can synergistically take full advantage of the mechanical property of each component as shown in Fig. $\mathrm{S} 5, \uparrow$ which may provide a potential method to prepare outstanding TSMPs with balanced mechanical properties.
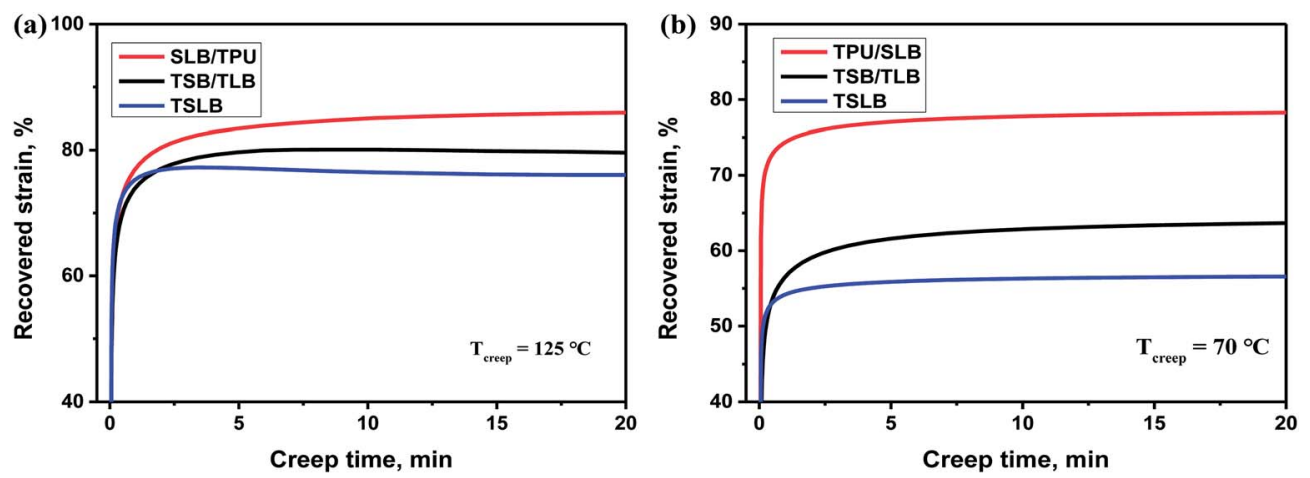

Fig. 9 Evolution of recovered strain of the specimens during the creeping progresses at (a) $125^{\circ} \mathrm{C}$ and (b) $70{ }^{\circ} \mathrm{C}$. 

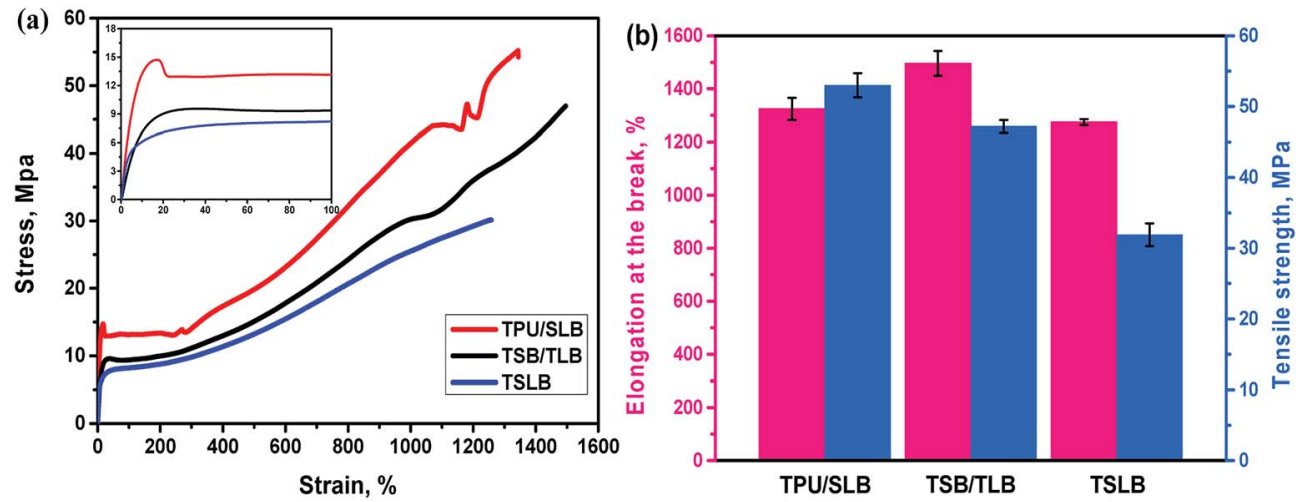

Fig. 10 (a) Stress-strain curves of the specimens (the inset shows the enlarged part of the curves); (b) comparison of tensile strength and elongation at break among the specimens.

\section{Conclusions}

TPU/PBS/PCL multicomponent materials with tunable phase continuity were designed and fabricated through layermultiplying co-extrusion. The well-separated $T_{\mathrm{m}}$ 's and $T_{\mathrm{c}}$ 's of PCL and PBS endow the specimens with capacity to achieve TSME. The results revealed that the multilayer systems possessing continuous phase and numerous interfaces have larger $R_{\mathrm{f}}$ and $R_{\mathrm{r}}$ than those of the blend. In particular, TPU/SLB with a special multi-continuous structure exhibits the best TSME because of the high phase continuity of each domain and stronger interfacial shearing effect. Besides, the multicontinuous structure maximally integrates the mechanical performances of the components, resulting in an excellent balance of $\sigma_{\mathrm{s}}$ and $\varepsilon_{\mathrm{b}}$. Hence, the current approach opens a new avenue for fabricating excellent TSMPs with optimal mechanical properties.

\section{Conflicts of interest}

There are no conflicts of interest to declare.

\section{Acknowledgements}

The authors are grateful to the National Natural Science Foundation of China $(51873132,51673136)$ for financial support of this work.

\section{References}

1 N. Zheng, Z. Z. Fang, W. W. Zou, Q. Zhao and T. Xie, Angew. Chem., Int. Ed., 2016, 55, 11421-11425.

2 Y. Q. Liu, K. G. Xu, Q. Chang, M. A. Darabi, B. J. Lin, W. Zhong and M. Xing, Adv. Mater., 2016, 28, 7758-7767.

3 Z. Li, X. Y. Zhang, S. Q. Wang, Y. Yang, B. Y. Qin, K. Wang, T. Xie, Y. Wei and Y. Ji, Chem. Sci., 2016, 7, 4741-4747.

4 G. H. Yang, X. J. Wan, L. J. Liu, R. Li, Y. K. Su, X. R. Zeng and J. N. Tang, ACS Appl. Mater. Interfaces, 2016, 8, 34744-34754.

5 J. K. Deng, Z. Y. Chang, T. Zhao, X. D. Ding, J. Sun and J. Z. Liu, J. Am. Chem. Soc., 2016, 138, 4772-4778.
6 H. Meng, J. Zheng, X. F. Wen, Z. Q. Cai, J. W. Zhang and T. Chen, Macromol. Rapid Commun., 2015, 36, 533-537.

7 J. J. Li, Y. H. An, R. Huang, H. Q. Jiang and T. Xie, ACS Appl. Mater. Interfaces, 2012, 4, 598-603.

8 M. Zarek, M. Layani, I. Cooperstein, E. Sachyani, D. Cohn and S. Magdassi, Adv. Mater., 2016, 28, 4449-4454.

9 Y. Huang, N. Zheng, Z. Q. Cheng, Y. Chen, B. W. Lu, T. Xie and X. Feng, ACS Appl. Mater. Interfaces, 2016, 8, 3562835633.

10 J. G. Hardy, M. Palma, S. J. Wind and M. J. Biggs, Adv. Mater., 2016, 28, 5717-5724.

11 Q. Zhao, H. J Qi and T. Xie, Prog. Polym. Sci., 2015, 49-50, 79120.

12 M. Behl and A. Lendlein, Mater. Today, 2007, 10, 20-28.

13 N. Zheng, J. J. Hou, Y. Xu, Z. Z. Fang, W. K. Zou, Q. Zhao and T. Xie, ACS Macro Lett., 2017, 6, 326-330.

14 V. Schimpf, B. Heck, G. Reiter and R. Mülhaupt, Macromolecules, 2017, 50, 3598-3606.

15 J. Du, D. B. Lu, Z. L. Zhang, X. L. Yao, D. C. Wan, H. T Pu and Z. Lu, Polymer, 2018, 126, 206-210.

16 L. Wang, X. F. Yang, H. M. Chen, T. Gong, W. B. Li, G. Yang and S. B. Zhou, ACS Appl. Mater. Interfaces, 2013, 5, 1052010528.

17 T. Xie, Nature, 2010, 464, 267-270.

18 C. Samuel, S. Barrau, J. M. Lefebvre, J. M. Raquez and P. Dubois, Macromolecules, 2014, 47, 6791-6803.

19 J. J. Li and T. Xie, Macromolecules, 2011, 44, 175-180.

20 X. F. Luo and P. T. Mather, Adv. Funct. Mater., 2010, 20, 26492656.

21 M. Behl, I. Bellin, S. Kelch, W. Wagermaier and A. Lendlein, Adv. Funct. Mater., 2009, 19, 102-108.

22 J. Zotzmann, M. Behl, D. Hofmann and A. Lendlein, Adv. Mater., 2010, 22, 3424-3429.

23 S. K. Ahn and R. M. Kasi, Adv. Funct. Mater., 2011, 21, 45434549.

24 K. Suchao-in and S. Chirachanchai, ACS Appl. Mater. Interfaces, 2013, 5, 6850-6853.

25 Q. W. Zhang, H. Q. Wei, Y. J. Liu, J. S. Leng and S. Y. Du, RSC Adv., 2016, 6, 10233-10241.

26 S. Chatani, C. Wang, M. Podgórski and C. N. Bowman, Macromolecules, 2014, 47, 4949-4954. 
27 T. Xie, Polymer, 2011, 52, 4985-5000.

28 J. L. Hu, Y. Zhu, H. H. Huang and J. Lu, Prog. Polym. Sci., 2012, 37, 1720-1763.

29 H. Zhang, H. T. Wang, W. Zhong and Q. G. Du, Polymer, 2009, 50, 1596-1601.

30 E. Kurahashi, H. Sugimoto, E. Nakanishi, K. Nagata and K. Inomata, Soft Matter, 2012, 8, 496-503.

31 S. M. Lai and Y. C. Lan, J. Polym. Res., 2013, 140, 2-8.

32 X. Jing, H. Y. Mi, H. X. Huang and L. S. Turng, J. Mech. Behav. Biomed. Mater., 2016, 64, 94-103.

33 H. J. Radusch, I. Kolesov, U. Gohs and G. Heinrich, Macromol. Mater. Eng., 2012, 297, 1225-1234.

34 S. M. Lai, P. Y. You, Y. T. Chiu and C. W. Kuo, J. Polym. Res., 2017, 24, 161.

35 J. Zhao, M. Chen, X. Y. Wang, X. D. Zhao, Z. W. Wang, Z. M. Dang, L. Ma, G. H. Hu and F. H. Chen, ACS Appl. Mater. Interfaces, 2013, 5, 5550-5556.

36 B. D. Favis and D. Therrien, Polymer, 1991, 32, 1474-1481.

37 C. E. Scott and C. W. Macosko, Polymer, 1995, 36, 461-470. 38 J. K. Lee and C. D. Han, Polymer, 2000, 41, 1799-1815.

39 W. L. Gao, Y. Zheng, J. B. Shen and S. Y. Guo, ACS Appl. Mater. Interfaces, 2015, 7, 1541-1549.

40 J. M. Zhu, J. B. Shen, S. Y. Guo and H. J. Sue, Carbon, 2015, 84, 355-364.

41 B. S. Chen, W. L. Gao, J. B. Shen and S. Y. Guo, Compos. Sci. Technol., 2014, 93, 54-60.

42 L. F. Yi, S. S. Luo, J. B. Shen, S. Y. Guo and H. J. Sue, ACS Sustainable Chem. Eng., 2017, 5, 3063-3073.
43 S. S. Luo, Y. Zheng, Z. Zheng, H. Wu, J. B. Shen and S. Y. Guo, Chem. Eng. J., 2019, 355, 710-720.

44 S. S. Luo, L. F. Yi, Y. Zheng, J. B. Shen and S. Y. Guo, Eur. Polym. J., 2017, 89, 138-149.

45 L. F. Yi, Y. Xu, D. Li, J. B. Shen, S. Y. Guo and H. J. Sue, Ind. Eng. Chem. Res., 2018, 57, 4320-4328.

46 J. Du, S. R. Armstrong and E. Baer, Polymer, 2013, 54, 53995407.

47 S. R. Armstrong, J. Du and E. Baer, Polymer, 2014, 55, 626631.

48 M. Michalak, M. Hakkarainen and A. C. Albertsson, ACS Sustainable Chem. Eng., 2016, 4, 129-135.

49 A. R. Costa-Pinto, V. M. Correlo, P. C. Sol, M. Bhattacharya, P. Charbord, B. Delorme, R. L. Reis and N. M. Neves, Biomacromolecules, 2009, 10, 2067-2073.

50 C. Zhang, X. Chen, G. T. Liu, H. Wu, J. Li, R. Chen and S. Y. Guo, RSC Adv., 2015, 5, 98999-99007.

51 Y. Deng and N. L. Thomas, Eur. Polym. J., 2015, 71, 534-546. 52 W. Klairutsamee, P. Supaphol and I. Jangchud, J. Appl. Polym. Sci., 2015, 132, 42716.

53 X. H. Wang, J. J. Zhou and L. Li, Eur. Polym. J., 2007, 43, 3163-3170.

54 M. F. Sonnenschein, S. J. Guillaudeu, B. G. Landes and B. L. Wendt, Polymer, 2010, 51, 3685-3692.

55 H. A. Khonakdar, S. H. Jafari, S. Rasouli, J. Morshedian and H. Abedini, Macromol. Theory Simul., 2005, 14, 428-434. 